\title{
Desarrollo agrario y complejidad económica: estudio de caso de una empresa agrícola de Murcia, España*
}

Cómo citar este artículo: Casaú Guirao, M. A., \& Noguera-Méndez, P. (20I7). Desarrollo agrario y complejidad económica: estudio de caso de una empresa agrícola de Murcia, España. Cuadernos de Desarrollo Rural, I4(80), I-I4. https://doi.org/ı0.III44/Javeriana.cdrı4-80.dace

\author{
Miguel Ángel Casaú Guirao \\ Universidad de Murcia, España \\ ORCID: http://orcid.org/0000-000I-7310-44I5 \\ Pedro Noguera-Méndez ${ }^{\text {a }}$ \\ Universidad de Murcia, España \\ pedrono@um.es \\ ORCID: http://orcid.org/0000-0003-2637-9920 \\ DOI: https://doi.org/I0.III44/Javeriana.cdrı4-80.dace \\ Redalyc: http://www.redalyc.org/articulo.oa?id=II756489007 \\ Recibido: $0_{9}$ Enero 2017 I Aceptado: 04 Agosto 2017
}

\section{Resumen:}

Desde la perspectiva de la complejidad económica se puede explicar el desarrollo a partir del proceso de acumulación de conocimiento incorporado a los bienes y servicios. A partir del estudio de la evolución de una empresa agrícola de Murcia (España), dedicada, desde 1995, a la producción, manipulación y comercialización de uva de mesa sin pepita y de su comparación con otras empresas agrícolas tradicionales, se examinan diversos conceptos de esta perspectiva; en particular la incorporación de conocimiento e información a los productos y la formación de redes tanto de personas como de empresas.

Palabras clave: desarrollo agrario, diversificación, complejidad económica, redes.

\footnotetext{
a Autor de correspondencia. Correo electrónico: miguelangel.casau@um.es
} 


\title{
Agrarian Development and Economic Complexity: Case Study of an Agricultural Company in Murcia, Spain
}

\begin{abstract}
:
From the perspective of economic complexity, development could be explained based on the process of knowledge accumulation incorporated into goods and services. We examine various concepts of this perspective based on the study of the evolution of an agricultural company in Murcia (Spain) that dedicates, since 1995, to the production, handling, and commercialization of seedless table grapes and their comparison with other traditional agricultural enterprises. Particularly, we focus on the incorporation of knowledge and information to the products and the creation of networks of both people and companies.
\end{abstract}

Keywords: agrarian development, diversification, economic complexity, networks.

\section{Introducción}

La complejidad, o la ciencia de los sistemas complejos, constituye una perspectiva metodológica aplicable al estudio de sistemas complejos adaptativos de muy diversas disciplinas (Benham-Hutchins y Clancy, 20ro; GellMann, 1988, 1995). La economía, desde la perspectiva de la complejidad, se entiende como un conjunto de sistemas adaptativos que coevolucionan y cuyo desarrollo depende del proceso de acumulación de conocimientos que una sociedad es capaz de incorporar a los bienes y servicios que produce. Las limitaciones que los individuos tienen para acumular conocimientos productivos se supera a través de las organizaciones y, a un nivel superior, con las redes de organizaciones o empresas (Hausmann, R. et al., 20I3; C. Hidalgo, 2015). La concepción de la economía como un sistema complejo adaptativo no sólo implica un cambio radical respecto a la perspectiva de la corriente principal de la economía (Beinhocker, 2006) sino que también ha demostrado ser un campo fecundo para encontrar respuestas nuevas a viejas preguntas sobre el desarrollo y la creación de la riqueza.

La comprensión del crecimiento y evolución de una economía, o de una empresa, puede mejorar cuando se considera su grado de complejidad. En particular se entiende mejor cómo el desarrollo económico, o el desarrollo de la agricultura, implica producir bienes distintos o de forma distinta, y no sólo producir más. La complejidad económica es directamente observable en el output productivo puesto que los tipos de bienes que una economía es capaz de producir muestran el grado de complejidad y la cantidad de conocimiento que es capaz de acumular. La complejidad de un producto viene directamente determinada por la cantidad y la combinación de información que requiere su obtención (Hausmann y Klinger, 2006; Hidalgo, Hausmann y Dasgupta, 2009). De manera que, expandiendo la acumulación de conocimiento, se llega a producir una mayor diversidad de bienes que sirve para poder desarrollar productos más complejos, que impulsan un crecimiento económico mayor (Hausmann, Cheston y Santos, 2015). Por tanto, la capacidad de formar redes es básica para elaborar productos más complejos puesto que los individuos tienen una capacidad para acumular información finita. La conformación de conjuntos de redes permite almacenar más información y, de esta manera, sostener el desarrollo de productos más complejos, y más sofisticados, que requieren de mayores estructuras de producción (Hidalgo, 2015).

Este trabajo pretende, además de presentar las ideas principales de la complejidad económica en relación con el desarrollo, cuestión a la que se dedica el apartado que sigue, realizar una aplicación de algunos de sus conceptos e ideas principales, al estudio de la evolución, a lo largo de veinte años, de una empresa agraria que ha tenido un comportamiento muy dinámico. La empresa española Sat Moyca, objeto del estudio, se dedica, desde 1995, a la producción, manipulación y comercialización de uva de mesa sin pepita. Haciendo uso de información primaria se examinará la empresa como una organización capaz de crear e incorporar valor a los productos. Su desarrollo ha tenido lugar en un entorno de interacción continua con elementos internos y externos diversos, que conforman un sistema complejo que, además, ha alcanzado una dimensión territorial global. Durante este período, no sólo ha crecido en términos de su dimensión productiva, sino que también lo ha hecho en términos del impacto de 
sus ideas e innovaciones. Este proceso se debe al incremento del conocimiento productivo incorporado, y a la formación de redes tanto de personas como de empresas.

En el estudio de caso se destacará la acumulación de conocimiento, sobre todo del tácito (“know-bow”, experiencia, habilidades...), cuyo papel es principal en el desarrollo y evolución de los productos. Se destacará asimismo el proceso de diversificación de la empresa, y sus consecuencias en términos de su estructura productiva y del nivel de sofisticación de sus productos. A partir de la información obtenida en las entrevistas, se han examinado las estructuras que definen las relaciones interempresariales de Moyca, por un lado, y las de una empresa tradicional, por otro, elaborándose sendas figuras que han servido para destacar sus diferencias y realizar un análisis comparativo de sus estructuras productivas y complejidad. En el último apartado se discuten los resultados, en particular en relación con sus implicaciones políticas y se presentan las conclusiones.

\section{Cambio productivo, diversificación y complejidad económica}

Desde la perspectiva de la complejidad, el desarrollo implica la emergencia de un sistema de instituciones económicas, financieras, legales, sociales y políticas, de empresas, productos y tecnologías que coevolucionan en un proceso adaptativo y que, conjuntamente, proveen a la sociedad de las capacidades para vivir con salud y felicidad vidas plenas (Barder, 20I2). El desarrollo, desde el punto de vista productivo, no sólo significa producir más sino sobre todo producir bienes y servicios diferentes, con más conocimiento incorporado, que permiten sostener una remuneración superior de los factores de producción (Hausmann et al., 2013; Hausmann y Klinger, 2006; Rodrik, 2005). Conviene destacar, en línea con el pensamiento de Prebisch y de la Cepal (Prebisch, 1986) que lo que se produce importa, que el desarrollo y las ganancias que un país pueda obtener de la globalización dependen crucialmente de lo que produce y, por tanto, exporta (Hausmann, Hwang y Rodrik, 2007). Además, es notable el proceso de diversificación productiva que los países experimentan con el desarrollo (Imbs y Wacziarg, 2003); aspecto que se destaca en el Atlas de la complejidad económica (Hausmann et al., 2013).

La obtención o fabricación de un producto requiere de conocimiento explícito (knowledge), de conocimiento tácito $(k n o w$-how) y de la conformación de redes, que pueden estar integradas por personas y organizaciones o empresas (Hidalgo, 2015). En su inspirador y original libro, César Hidalgo explica con detalle y ejemplos que el conocimiento y el know how son capacidades fundamentales para la acumulación de información en la economía y en la sociedad. Pero no son la misma cosa. Mientras que el conocimiento implica relaciones o vínculos entre entidades, y ayuda a entender estas relaciones, el know-bow "consiste en la capacidad de llevar a cabo acciones, aun sin explicárselo" (Hidalgo, 2015, p. I4), debido a su naturaleza tácita.

La capacidad que tiene un individuo de acumular conocimiento es limitada y se puede denominar personbyte. A través de la interacción de los individuos en redes más complejas se amplía sensiblemente esa capacidad: las redes $\mathrm{u}$ organizaciones de individuos en empresas $\mathrm{u}$ organizaciones (firmbyte) $\mathrm{y}$, a un nivel superior, redes de empresas (globalbyte) (Hidalgo, 2015).

Por su parte, el output productivo expresa la complejidad económica de los sistemas productivos, pues la diversidad y la calidad de los bienes que es capaz de producir un país refleja la cantidad de conocimiento útil que hay en esa economía. Simultáneamente, para que esa cantidad de conocimiento sea acumulado, debe haber una amplia red de personas que puedan generar, de forma conjunta, diversos productos (Hausmann et al., 20I3). 
Ese desarrollo se lleva a cabo de manera gradual, saltando las empresas de unos productos a otros que son próximos, en la medida en la que estas comparten determinados activos, recursos, instituciones y conocimientos necesarios para producirlos. Por ejemplo, si una empresa es capaz de fabricar camisetas, es más probable que pueda también fabricar camisas o chaquetas en lugar de electrodomésticos o productos informáticos, ya que hay más interrelación entre ellos y las capacidades técnicas, humanas o institucionales que se necesitan para fabricarlos son más próximas a las necesarias para desarrollar dichos artículos. En consecuencia, la diversidad de productos que una economía puede producir depende directamente de la diversidad de capacidades que tiene (Hidalgo et al., 2007).

De esta manera, se definen trayectorias de especialización productiva que resultan más probables que otras, y que implican el tránsito de una oferta basada en bienes más simples a otra en la cual se encuentran presentes los bienes con mayores requisitos y conocimientos incorporados (Hausmann y Klinger, 2006). Así, las economías se diversifican, pues se amplía el número de productos, y estas se capacitan para producir bienes más exclusivos, en el sentido de que un menor número de países es capaz de producirlos. En ese sentido, la diversificación de la oferta productiva y la menor ubicuidad de los bienes que se producen son características de la complejidad. Además, precisamente esos conceptos de diversidad y ubicuidad les sirven a los autores del Atlas del espacio del producto para calcular la complejidad económica de países y de productos.

Entendemos que la economía de la complejidad se interesa por la cantidad de conocimiento productivo que tiene una economía, que es difícil de transferir y acumular. Se puede asumir que ese conocimiento productivo se divide en lo que llamamos capacidades, que son los requisitos de producción de los bienes. Pero esa acumulación presenta numerosas dificultades, puesto que una economía no puede fabricar productos que requieran capacidades determinadas que no se encuentren en el territorio, entonces, será más probable que una economía fabrique productos que requieran de capacidades que ya existen en los productos que ya sea capaz de crear y, por tanto, irá produciendo otros bienes cuyos requerimientos impliquen un conjunto de conocimientos y capacidades similares o próximos a los incorporados en los productos ya existentes (Hausmann et al., 2013).

El espacio del producto, que es una de las contribuciones centrales del Atlas de complejidad económica (Hausmann et al., 2013), es una representación de la red que conecta a los productos en función de la probabilidad de que sean coexportados y puede ser utilizado para predecir la evolución de la estructura exportadora de un país.

El espacio del producto muestra el grado de similitud que hay entre los bienes a través de un mapa de redes. Cuando dos productos están 'próximos' entre sí en el Espacio del Producto, su similitud es alta, queriendo esto significar que los activos, recursos, instituciones y capacidades requeridos para la obtención de un bien son aplicables, al menos parcialmente, a la producción del otro bien. Si un bien tiene muchos otros productos 'cerca' o próximos, quiere decir que las capacidades que el país tiene para producir un bien determinado podrían contribuir a producir otros bienes, diversificando así la oferta productiva. Si, por el contrario, la especialización productiva se concentra en bienes situados en la periferia del espacio del producto, que son zonas de baja densidad, las posibilidades de diversificar la oferta subiendo por la escalera de la tecnología y de la productividad serán menores. En general, los productos más complejos se sitúan en zonas altamente densas y mantienen un elevado número de conexiones con otros productos, mientras que los menos complejos estarían en las zonas más periféricas, manteniendo un menor número de conexiones (Hausmann y Klinger, 2006; Hidalgo, Klinger, Barabási y Hausmann, 2007).

En ese escenario, la proximidad productiva con respecto a una amplia variedad de bienes es un aspecto clave para el proceso de transformación que permite diversificar una oferta de mayor productividad; en otras palabras, de bienes más sofisticados y capaces de otorgar mayores remuneraciones con respecto a los factores que comprometen. Asimismo, el proceso de transformación productiva ocurre con mayor probabilidad siguiendo las trayectorias determinadas por la especialización existente, en un continuo proceso de adaptación, combinación y ampliación de capacidades, que permite saltar a productos próximos mediante la acumulación de conocimientos. La evolución descrita implica un proceso de aprendizaje que está sesgado, en el sentido de que la posición inicial, la cual es 
definida por la oferta productiva existente, importa, condicionando su evolución (Hidalgo, 2015; Hidalgo et al., 2009).

En ese escenario, la división del trabajo permite la fragmentación del conocimiento, mientras que la sociedad puede movilizar, por medio de las redes, enormes cantidades acumuladas de conocimiento. Es decir, la acumulación de conocimiento no depende de la cantidad limitada de conocimiento de cada individuo, de cada empresa u organización sino de la capacidad que alcance en conjunto, como sociedad, para formar redes en las que se utilice y recombine el conocimiento (Hausmann et al, 2013).

A la capacidad máxima de conocimiento que un individuo es capaz de retener Hidalgo (2015) la denomina personbyte. En términos de complejidad, el crecimiento de conocimiento productivo y el desarrollo de redes están directamente relacionados. Como la capacidad del individuo de almacenar conocimiento es limitada, para desarrollar bienes más complejos, y que necesiten para fabricarlos más de un personbyte, se requiere de otros individuos (organizaciones o empresas) que formen redes. La capacidad de acumular conocimiento de estas redes también es limitada y se cuantifica a través del firmbyte de manera que para aumentar más la cantidad de conocimiento que se puede acumular se deben formar redes de empresas. Ambos conceptos (personbyte y firmbyte) representan unos límites conceptuales que implican una necesidad de formar la estructura de las redes necesaria para acumular conocimiento (Hidalgo, 2015). Pero existen factores que limitan la formación de esas redes. Una teoría que habla de esos límites es la "teoría de los costos de transacción" de Coase (1937) que indica la existencia de una relación inversa entre los costos de formar vínculos y el tamaño (complejidad) de las redes que se pueden formar. Es decir, cuanto mayor sea el coste, el tamaño de las redes estará más limitado, mientras que si los costes son más bajos se podrán construir redes muy grandes. El tamaño limitado de las empresas indica que si el volumen de conocimiento necesario es más grande que las redes que podemos formar será necesario compartirlo formando redes de empresas (globalbyte) (Hidalgo, 2015).

En consecuencia, la capacidad computacional vendrá determinada por la capacidad de formar redes; es decir, por el capital social. El capital social se entiende como la habilidad que tienen los individuos para formar redes con las que se puede acumular un mayor número de personbytes; un aspecto que responde a la idea de que las relaciones sociales tienen un valor económico. El capital social es importante ya que, mediante su delimitación, es posible explicar cómo se produce crecimiento económico, puesto que la necesidad de formar redes viene dada por la habilidad limitada de los individuos de acumular conocimiento. Al formar redes, las personas son capaces de almacenar una mayor cantidad de conocimiento que les permite mejorar las habilidades propias para procesar información y producir bienes (Hidalgo, 2015).

Entonces, la esencia del crecimiento económico se encuentra en el crecimiento de la información en una economía, que es el resultado de la evolución de la capacidad computacional y de la acumulación de conocimiento de los individuos al formar redes cada vez mayores que permiten desarrollar productos que, partiendo de los existentes, permitan producir bienes más complejos (Hidalgo, 2015).

\section{Metodología}

\section{La información}

El método de investigación aplicado es el estudio de caso (Martínez Carazo, 2006) de la empresa agraria productora de uva de mesa Moyca, ubicada en la región de Murcia (España). Se ha recopilado información sobre sus características y evolución, lo que incluye información sobre sus precios y sobre sus actividades. La entrevista en 
profundidad a un miembro fundador de la empresa y actual propietario constituye una parte fundamental de la información disponible. Además, se entrevistó a otro socio fundador de la empresa y los servicios administrativos de la empresa proporcionaron informaciones diversas. La entrevista se ha realizado sobre una base de preguntas con respuesta abierta que fue grabada, transcrita y objeto de un exhaustivo análisis.

En las entrevistas y en los datos recopilados, se han considerado cuatro dimensiones básicas. En primer lugar, se ha tomado en consideración la información general disponible sobre la evolución que ha registrado la empresa desde su creación, en 1995, hasta la actualidad; en consecuencia, se toman en cuenta aspectos como las instalaciones, el volumen de producción y el número de trabajadores, etc. En segundo lugar, se toma en consideración la información relativa a su estructura interna, con el objetivo principal de conocer cómo ha evolucionado el proceso de incorporación de información a la empresa. En tercer lugar, ha sido de interés conocer el output productivo de la empresa, puesto que, tal y como se ha indicado anteriormente, este expresa el grado de complejidad que tiene una economía; de tal manera, se examinará qué productos es capaz de elaborar: su diversidad, exclusividad y evolución. Lo anterior permitirá conocer cómo la empresa ha evolucionado añadido conocimiento a su estructura productiva. Por último, se examina la evolución de la formación de redes de empresas de Moyca con otras firmas o instituciones para ser capaz de adquirir más conocimiento productivo. De esta forma, se podrán conocer las colaboraciones que la compañía ha desarrollado con otras empresas e instituciones, las cuales implican el tejido de redes con ellas; operaciones organizadas con la finalidad de producir y desarrollar nuevos tipos de productos. Respecto a esto conviene destacar la diferenciación establecida entre las relaciones mantenidas con las empresas hibridadoras, por un lado, y con las empresas productoras de uva de mesa sin semilla, por otro. De este modo, se podrá conocer mejor la capacidad de formar redes y, por tanto, el potencial para acumular una mayor cantidad de conocimiento productivo.

Finalmente, con base en las entrevistas, de informaciones de expertos del sector y de fuentes secundarias, se han elaborado sendas imágenes (Figura 2) que representan las relaciones productivas de Moyca y de una empresa de uva de mesa tradicional. En estas figuras se pretende mostrar el marcado contraste que existe, en cuanto a complejidad económica, entre ambos tipos de empresas.

\section{El análisis de los datos}

El análisis de los datos permitirá interpretar rasgos como la evolución, las características, el crecimiento y los resultados de la empresa, en términos del marco teórico descrito anteriormente. Con ese propósito, la información ha sido clasificada, y se ha procedido a desarrollar su análisis, en la medida en que se consideran cinco ámbitos principales que atienden las necesidades de este trabajo de investigación. Según lo expuesto, el análisis en profundidad de la información permite estudiar la evolución de la empresa desde la perspectiva de la complejidad económica, con lo que se comprueba el protagonismo que han tenido las redes, la información y los procesos de acumulación de conocimiento. Estos ámbitos son:

(i) El crecimiento de la empresa.

(ii) El aumento de la información en la estructura interna.

(iii) El aumento de la información en el producto.

(iv) La capacidad de formar redes.

(v) La comparación de las redes de relaciones productivas de Moyca y de la empresa de uva de mesa tradicional. 


\section{Resultados}

\section{Crecimiento de la empresa}

La empresa Moyca ha experimentado un intenso crecimiento, pues ha pasado de ser una pequeña empresa familiar agrícola dedicada a la venta de uva de mesa, a transformarse en una de las empresas más importantes del sector de la uva de mesa de España. Tal dinámica ha implicado un fuerte flujo inversor, y una creciente demanda de trabajo. En términos de producción, lo anterior ha significado pasar de una cantidad inferior al millón de kilogramos en 1995, a cifras que superan los cuarenta millones de kilogramos de uva al año en 20r6; una evolución que ha acarreado el consiguiente aumento de los terrenos para cultivar, que han pasado de comprender cincuenta hectáreas a ser más de mil cien: un resultado que se ve reflejado en sus centros de manipulación -lugares en donde se empaqueta la uva y se distribuye a los supermercados-, pues estos han pasado de i a 4. En términos del empleo, su evolución es también sobresaliente, pues de generar veinte empleos fijos y cincuenta temporales en el año 1995, se ha pasado a mantener una plantilla de cuatrocientos trabajadores fijos, mientras que se proveen tres mil empleos temporales durante la época de cosecha y producción.

\section{Aumento de la información en la empresa}

Ante los parámetros expuestos, la figura i describe los sucesivos cambios que han sucedido con relación a la organización interna y a los objetivos de la empresa. Según tales disposiciones, no solo ha sido sobresaliente el crecimiento de esta empresa, sino también su evolución y su adaptación a un proceso continuo de innovación y cambio que ha requerido la acumulación de la información y el conocimiento que han sido incorporados al producto. En el año 2007, Moyca registró un relevante cambio jurídico, puesto que en ese momento pasa de ser una empresa familiar a constituirse como una sociedad agraria de transformación (SAT) que se integra a una organización de productores de frutas y hortalizas (OPFH).

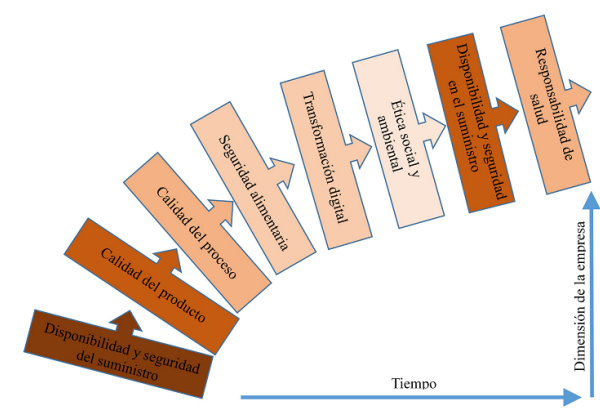

FIGURA 1

EvOLUCIÓN Y CRECIMIENTO DE LA EMPRESA

FUENTE: ELABORACIÓN PROPIA

En efecto, la evolución y el crecimiento de la empresa aparecen marcados por la progresiva y creciente consideración de objetivos, así como por la incorporación de información (Figura I). En un primer momento, en el que se ha garantizado previamente la estabilidad y la continuidad de la producción, se otorgan mejoras a la calidad del producto por medio del surgimiento de nuevas variedades y el perfeccionamiento de las existentes, lo que conduce a la compañía a diferenciarse de sus competidores. Posteriormente, se mejoran los procesos de producción, en tanto que la madurez de la empresa permite la consideración de otros aspectos y dimensiones que actualmente 
son básicos en el marketing: se alude así a la mayor preocupación por la seguridad alimentaria; a la adaptación a los nuevos hábitos alimenticios de la población; a la transformación digital que permite a la empresa estar presente en las redes sociales y obtener un mayor acceso a la información por parte de clientes y proveedores, al igual que a los cambios de los procesos productivos que son más respetuosos con el medio ambiente. Con la inclusión, al final de la figura i de la "Disponibilidad y seguridad del suministro" - alusión dada por segunda vez en todo el texto-, se destaca que, a partir de 2015 , la empresa enfoca sus prioridades en la cantidad y el desarrollo del suministro, tal y como sucedía en la primera fase; sin embargo, en ese contexto, dicho suministro aumenta en términos de cantidad y variedades, pues participa directamente en programas de hibridación. En la actualidad, mientras se sostienen todos los objetivos expuestos previamente, la prioridad se sitúa en la realización de nuevas mejoras en los productos de la compañía, para que las uvas atiendan las demandas de salud que son propias de los mercados.

\section{Aumento de la información en el producto}

El aumento de la información del output productivo refleja el grado de complejidad de una economía (Hidalgo, 2015). En ese escenario, un cambio fundamental del output productivo de Moyca consiste en el paso de producir uva de mesa con pepita, a proveer uva de mesa sin pepita; transición que significó, primero, una especialización en este producto, lo cual condujo a registrar después una intensa diversificación, pues se producen hasta treinta variedades diferentes de uva sin semilla. Estas variedades están englobadas en seis grandes tipos: (i) las variedades rojas sin semillas, que representan más del 50\% del output, seguidas de (ii) las verdes; en tanto, con participaciones sensiblemente inferiores, se encuentran (iii) las negras y (iv) las mixtas; y, por último, (v) las variedades especiales, y (vi) las orgánicas.

Existe una analogía entre el proceso de diversificación productiva, que se describe en el marco del espacio del producto (Hausmann et al, 2013), y el desarrollo de nuevas variedades de uva, pues éste se lleva a cabo a través del cruce de las variedades ya existentes, hasta que se encuentren con una variedad que sea de mayor calidad y mejor que las actuales. Actualmente, existe una preocupación por la mejora de las variedades existentes, que es postulada con la finalidad de que los productos aporten beneficios para la salud como, por ejemplo, que tenga mayores niveles de calcio o de selenio, o que los cultivos que las proveen sean orgánicos, o estén libres de productos fitosanitarios y pesticidas. Estas variedades especiales, así como las orgánicas, que tienen un mayor valor añadido, van a experimentar un crecimiento relativo en el output productivo en los próximos años. Esta evolución, que ha implicado la multiplicación de variedades y la búsqueda de variedades especiales que permiten satisfacer propósitos determinados (sabor, color, salud, etc.) se ha basado en un proceso de acumulación de conocimiento en la empresa que se ha aplicado a los productos. La mejora en los productos ha significado asimismo su mayor complejidad y el aumento de la información y del conocimiento incorporado tanto en los productos como en el conjunto de la empresa.

Así, la diferenciación descrita del producto, la cual incorpora más conocimiento y más información, implica asimismo una mayor remuneración de los factores productivos; un mayor valor añadido y, en consecuencia, un mayor precio, tal y como lo refleja la información presentada en la tabla i. En efecto, teniendo como excepción el primer año, los precios que perciben los agricultores por las uvas apirenas superan sensiblemente los percibidos por las uvas con semillas; se constituyen así diferencias que alcanzan el $35 \%$, en 20 II, y el $29 \%$ en 2015 (ver la penúltima fila de la Tabla i). Cabe añadir que las diferencias mencionadas se amplían cuando se toman en consideración los valores correspondientes al siguiente escalón de la comercialización: aspecto que corresponde a los precios que tienen los productos cuando salen del almacén. En este caso, cuando se comparan ambas series de precios destaca la superioridad de los precios de la uva apirena, que alcanza su máximo en el año 2007 con el 64\%, mientras que en el año 2015 el precio de este tipo de fruto es un $32 \%$ mayor que el de la uva con semillas (ver la última fila de la Tabla I). 
TABLA 1

Precios percibidos por los Agricultores y precios Al SAlir del Almacén de las uvas apirenas y CON SEMILlas. $2006-2015$

\begin{tabular}{|l|r|r|r|r|r|r|r|r|r|l|}
\hline Euros / kg & $\mathbf{2 0 0 6}$ & $\mathbf{2 0 0 7}$ & $\mathbf{2 0 0 8}$ & $\mathbf{2 0 0 9}$ & $\mathbf{2 0 1 0}$ & $\mathbf{2 0 1 1}$ & $\mathbf{2 0 1 2}$ & $\mathbf{2 0 1 3}$ & $\mathbf{2 0 1 4}$ & $\mathbf{2 0 1 5}$ \\
\hline $\begin{array}{l}\text { Precios del } \\
\text { agricultor }\end{array}$ & & & & & & & & & & \\
\hline Apirenas & 0,48 & 0,68 & 0,72 & 0,62 & 0,70 & 0,74 & 0,62 & 0,66 & 0,75 & 0,72 \\
\hline Con semillas & 0,52 & 0,55 & 0,61 & 0,57 & 0,61 & 0,55 & 0,55 & 0,63 & 0,72 & 0,56 \\
\hline Precios del almacén & & & & & & & & & & \\
& & & & & & & & & & \\
\hline Apirenas & 1,18 & 1,44 & 1,38 & 1,35 & 1,44 & 1,47 & 1,47 & 1,41 & 1,39 & 1,58 \\
\hline Con semillas & 0,86 & 0,88 & 1,00 & 0,95 & 1,02 & 0,99 & 1,23 & 1,08 & 1,10 & 1,20 \\
\hline $\begin{array}{l}\text { Precio de apirenas / } \\
\text { Precio con semillas* }\end{array}$ & & & & & & & & & & \\
\hline Agricultor & 0,92 & 1,24 & 1,18 & 1,09 & 1,15 & 1,35 & 1,13 & 1,05 & 1,04 & 1,29 \\
\hline Almacén & 1,37 & 1,64 & 1,39 & 1,43 & 1,42 & 1,49 & 1,20 & 1,30 & 1,26 & 1,32 \\
\hline
\end{tabular}

Fuente: elaboración propia a partir de la información proporcionada por el Servicio de Estadística de la Consejería de Agricultura y Agua de la Región de Murcia (http://www.carm.es).

Nota: para el cálculo de estos promedios, no se han tomado en consideración las variedades cuyos precios no están disponibles durante la mayoría del período. * Cociente entre el precio de las uvas apirenas y el precio de las uvas con semillas, calculado para los agricultores y a la salida de almacén.

\section{Capacidad de formar redes}

Las redes de empresas que Moyca ha tejido constituyen algunos de los aspectos más relevantes para explicar su crecimiento, puesto que le han permitido desarrollar nuevos productos y mejorar su distribución en el mercado. Cabe señalar que Moyca tiene estrechas relaciones con las empresas hibridadoras o breeders. Estas empresas consiguen, a partir de cruces entre variedades de uva, otras variedades con características diferentes y de mayor calidad. Moyca produce y comercializa estas nuevas variedades que ofrecen al mercado calidad y novedad. La relación entre Moyca y las empresas hibridadoras ha evolucionado. Al inicio de su relación, Moyca se limitaba a adquirir la correspondiente variedad al breeder, para producirla y comercializarla. La relación se ha intensificado con los breeders hasta el punto de ser socias o participar en los propios proyectos de desarrollo de nuevas variedades de uva. Por ejemplo, Moyca es miembro, desde el año 2005, de la Sociedad Murciana de Investigación y Tecnología de Uva de Mesa S.L., (ITUM), que colabora asimismo con el Instituto Murciano de Investigación y Desarrollo Agrario y Alimentario (Imida). También encontramos que Moyca ha adquirido licencias con otros breeders como SNFL group con la que produce io variedades distintas, con International Fruit Genetics I2 variedades, y también con Sun World o Arra Varieties.

Además, la formación de redes de empresas para el intercambio de información es fundamental para el funcionamiento del sector. De hecho, de acuerdo con la información recogida, algunos cambios registrados en el sector obedecen a su necesaria adaptación para el adecuado funcionamiento y la supervivencia en los mercados. En efecto, anteriormente, las nuevas variedades desarrolladas por las empresas hibridadoras se subastaban, de modo que las adquiría el mejor postor. Sin embargo, tal proceder no garantizaba su producción, pues el comprador no tenía por qué tener la capacidad técnica ni el know-how necesarios; en consecuencia, aunque se hubiese desarrollado una variedad mejor que las existentes, esta no siempre se vendía, porque la empresa que adquiría los derechos de producción podía no tener las capacidades computacionales y técnicas para producirla y comercializarla. Posteriormente, ante el fracaso de muchos de esos procesos, las empresas hibridadoras cambiaron sus métodos; de ese modo, con el fin de garantizar que sus variedades se comercializaran, ofreciéndolas solamente a empresas con acreditada capacidad de producción. 
Como la producción de uva es estacional, mientras que el consumo es un factor mucho más estable, los países exportadores, por lo general, no tienen las condiciones idóneas para producir durante todo el año, por lo que deben importar las uvas. Por esta razón, la distribución moderna, como la que ocurre en las cadenas de supermercados, precisa una oferta diversificada de proveedores de diferentes regiones del mundo que permita garantizar la presencia del producto en las cadenas lineales correspondientes. No obstante, la variedad de uvas disponible debería ser la misma, por lo que en distintos países del globo las empresas del sector intercambian información técnica para así poder exportar la misma variedad de uva a lo largo de todo el año. Así, Moyca transmite esa información a empresas de países de Sudáfrica, Egipto o Chile con que, tanto la compañía como el conjunto de las demás empresas, mejoran sus capacidades técnicas al compartir diversos métodos y experiencias de producción. Se crean entonces redes internacionales entre Moyca, otras firmas y los breeders, lo cual se permite producir y comercializar distintas variedades de uva.

\section{Mapa comparativo de relaciones productivas de las redes}

El mapa elaborado de las redes de Moyca y de la empresa productora tradicional revela la existencia de diferencias notables entre los respectivos procesos y relaciones productivas. Se pueden indicar cuatro fases del proceso: (i) la tecnología con la que se desarrollan las variedades de uva; (ii) la producción en donde se plantan, cosechan y cortan;(iii) la distribución en los almacenes, en donde se empaquetan los frutos; y (iv) la posterior comercialización en los supermercados u otros establecimientos.

La primera etapa, identificada en la figura 2 con el término tecnología, consiste en la compra por parte de los productores de variedades de uva licenciadas que desarrollan las empresas hibridadoras o breeders. En esta fase radica la principal diferencia con la empresa tradicional, puesto que, con base en esas relaciones con el sector tecnológico - representado tanto por breeders como por otros productores-, Moyca incorpora conocimientos a su estructura productiva. Una vez que las variedades han sido adquiridas, los productores de distintas regiones geográficas intercambian información para coordinar sus procesos productivos, de manera que puedan producir la misma variedad de uva, fruto que cuenta así con las mismas características, con el fin de atender a la linealidad del consumo. Los productores cosechan en viveros y plantaciones de prueba el material adquirido de parte de los hibridadores, para así verificar las condiciones óptimas y la recepción del mercado; cuando se completa este proceso, se realiza la plantación masiva en las cantidades necesarias. Posteriormente, el producto se cosecha, se empaqueta y se prepara para su distribución. Finalmente, la uva se vende a las distintas cadenas de supermercados.

La descripción del proceso productivo y de las relaciones de una empresa de uva de mesa tradicional se muestra en la parte inferior de la Figura. La tecnología es mucho más limitada ya que la capacidad de aprendizaje que tiene una sola empresa de acumular información y conocimiento es mucho menor que en el caso anterior donde se han formado redes. La variedad de uva que vende la empresa tradicional es libre, es decir, no licenciada y, por tanto, puede o bien comprarla a un productor, en el caso de no tener capacidad de producción, envasarla y venderla o él mismo cosecharla en la plantación sin necesidad de unas plantaciones de prueba, llevarla al almacén para envasarla y finalmente proceder a su distribución. Pero la diferencia no sólo se encuentra en el producto, también en el destino de sus ventas puesto que en lugar de distribuir el producto a cadenas de supermercados donde hay que cumplir unos mayores estándares de calidad, sus ventas se suelen realizar en otros establecimientos como las lonjas, las tiendas o los discounts o supermercados de "segunda división". Por tanto, el limitado tamaño de las redes también limita el proceso de distribución pues al no poder incorporar tanta información al producto, su distribución no alcanza a las grandes cadenas de supermercados, sino a comercios de menores dimensiones. 

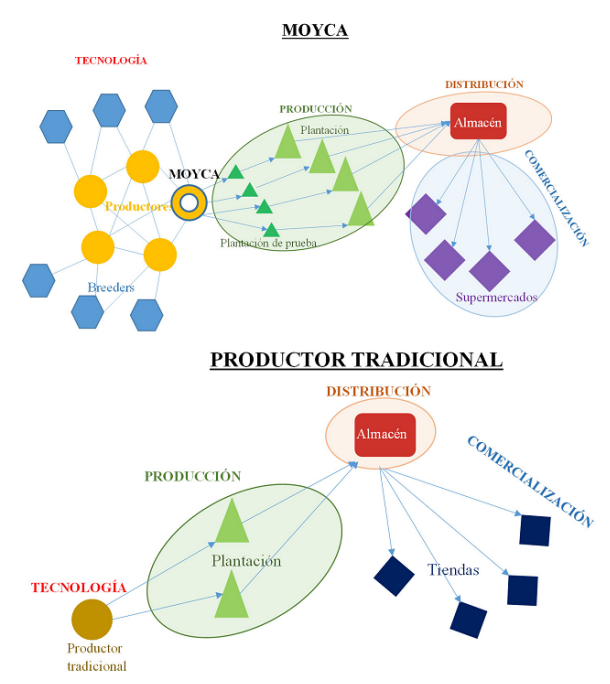

FIGURA 2

Mapa de Redes de Moyca y de una empresa tradicional FuENTE: ELABORACIÓN PROPIA

\section{Discusión y conclusiones}

A partir del análisis de la información y de la comparación de las estructuras productivas de Moyca y de la empresa 'tradicional' del sector, se ha alcanzado el propósito de examinar el crecimiento de la empresa Moyca desde la perspectiva de la complejidad económica, observando cómo ha aumentado la información en la propia empresa y en los bienes producidos, así como su capacidad de formar redes. Desde este punto de vista se debe estudiar el papel del conocimiento, su evolución en la empresa y cómo esto se expresa en su output productivo. La consideración de todas estas cuestiones es la que posibilita la aproximación a la complejidad en la empresa.

La evolución de Moyca evidencia un crecimiento sobresaliente, tanto en lo que atañe a la producción, como en lo relativo a los tipos de productos y el número de trabajadores. En el año 2007, la empresa cambia su régimen jurídico, pues pasa de ser una empresa familiar a consolidarse como una sociedad agraria de transformación (SAT), integrada simultáneamente a una organización de productores de frutas y hortalizas $(\mathrm{OPFH})$. Este nuevo régimen jurídico permite acceder a ayudas de la Unión Europea para financiar inversiones y mejoras en lo que respecta a medios técnicos y almacenaje, lo cual en general representa un incentivo para la mejora de las empresas del sector. Es decir, conforme ha ido creciendo, Moyca se ha adaptado, incluso jurídicamente, lo que le ha permitido a la compañía aprovechar ciertas ventajas.

La diferenciación que hay entre los dos tipos de conocimiento -el explícito y el tácito- los cuales fueron explicados en el marco teórico, al igual que el modo como estos influyen sobre la estructura productiva, pueden examinarse con base en la información recogida en las entrevistas; pesquisa en la cual los interlocutores de la empresa insisten en la importancia del know-bow o conocimiento tácito. Por un lado, cuando un productor compra los derechos para producir una variedad determinada a la empresa hibridadora, necesita de ese know-bow, experiencia o capacidad técnica, para poder producirla de manera correcta y estable puesto que, de no ser así, esa variedad, que presenta determinadas 'mejoras' respecto a las ya implantadas y, por ello, es previsible su éxito en el mercado, fracasa. Por otro lado, para que de una variedad pueda existir una producción regular y continua que garantice su presencia en los supermercados, debe haber un intercambio de conocimiento productivo entre varios productores de distintas zonas geográficas que permita que, finalmente, esta se comercialice de manera efectiva, ofreciendo las mismas características y la misma calidad, aunque tenga diversas procedencias. Por tanto, no solo es necesario que se utilice el conocimiento explícito - caracterizado por su fácil codificación y transferencia- para desarrollar un 
nuevo producto, sino que para que pueda funcionar comercialmente el nuevo producto, o la nueva variedad en este caso, se debe realizar un intercambio de experiencias, de capacidad técnica y de conocimiento productivo.

El proceso de especialización, rasgo fundamental según la perspectiva de la complejidad económica, constituye una dimensión muy importante de la evolución registrada en Moyca. En este caso, Moyca ha visto una oportunidad de negocio que se podía explotar en el mercado europeo; en consecuencia, adopta la decisión de dedicarse exclusivamente a la producción, comercialización y distribución de uva de mesa apirena o sin semilla.

La diversificación llevada a cabo por Moyca se ha basado en el desarrollo de bienes similares a los que ya producía, es decir: ha producido variedades nuevas de uva con base en las ya existentes y en las capacidades, conocimientos y otros activos disponibles, encajando tal dinámica en la prevista por la perspectiva del espacio del producto (Hausmann et al, 2013). Según ese marco operativo, la diversificación ocurre con mayor probabilidad aplicando las capacidades existentes - como los activos, los recursos y la experiencia-determinadas por lo que se produce, a la producción de nuevos bienes. En este sentido, cuando una empresa hibridadora vende los derechos de una nueva variedad a Moyca, lo hace porque tiene garantías de la capacidad técnica y el know-how de MOYCA, necesarios para producir su variedad.

En suma, el esfuerzo de la empresa por mejorar su capital social y, por tanto, su capacidad de formar redes, ha tenido un papel fundamental en su crecimiento; gracias a eso, la firma ha conseguido elevar su capacidad computacional y aumentar el grado de conocimiento que se agrupa en ella. Tal y como se ha indicado anteriormente, las redes permiten superar las barreras que limitan la acumulación de conocimientos: en consecuencia, redes más grandes, bien sea de individuos o de empresas, pueden llevar a cabo procesos de producción más sofisticados (Hidalgo, 2015).

Es decir, el intercambio de información comienza en el primer momento en que hay contacto entre las empresas de distribución -el mercado-y la propia empresa. Cuando Moyca decide redireccionar toda su producción hacia la uva de mesa sin pepita, pues percibe que hay una oportunidad de mercado, -según se puede interpretar- existe un intercambio de información entre los consumidores y la empresa; intercambio por el que ésta se transforma, adaptándose a la demanda potencial, gracias a la computación de esa información. Pero para poder computar y aplicar a los procesos esa información, es necesaria una mayor capacidad para formar redes de parte de Moyca, puesto que las grandes redes son las que permiten grandes acumulaciones de conocimiento.

La empresa comienza estableciendo relaciones con los breeders cuando decide producir uva de mesa licenciada sin pepita, que es más sofisticada que la que sí tiene pepita, que producía y comercializaba anteriormente. Este cambio implica que para poder producir las nuevas variedades, que son productos más complejos, tiene que formar redes con las empresas hibridadoras (Figura 2). Estas empresas, a través de cruces de distintos tipos de uva desarrollan variedades nuevas, mejores o distintas que las existentes, y Moyca la que, comprando los derechos, la produce, distribuye y comercializa. La especialización también concierne al reparto de funciones y de conocimientos de breeders y productores, que forman redes que permiten aumentar la capacidad de cómputo y desarrollar las nuevas variedades de uva, aumentar el output productivo y la complejidad. Además, con el fin de poder comercializar con éxito una variedad de uva registrada de forma estable y permanente, debe formar redes con otras empresas productoras, intercambiar información técnica y establecer una planificación que asegure la coordinación. Las redes de productores y breeders también alcanzan a la investigación puesto que, en el caso de Moyca, la empresa participa activamente en los proyectos de investigación de diversos breeders llevando a cabo líneas conjuntas de desarrollo de nuevas variedades de uva, como en el caso de Itum del que Moyca es socia junto a otros productores de uva de mesa apirena, o con International Fruit Genetics con la que, aunque no es socia, participa en proyectos de investigación e hibridación de doce variedades licenciadas de uva.

Los intensos procesos de diversificación y especialización, así como la capacidad de formar redes de Moyca, han constituido factores claves para el incremento de información y conocimiento del output productivo de la empresa. Es esencial recordar, en lo referido a ese aspecto, que Moyca comenzó sus actividades con el cultivo y 
gestión de la uva de mesa con pepita, un producto no diferenciado, estandarizado, que contaba con escaso valor añadido y cuya producción exige unos bajos y accesibles requerimientos. "El acceso al mercado era muy fácil para todos" (Comunicación personal, 20 de abril de 2017), indica un interlocutor de la empresa, lo cual significa que el producto podía calificarse como altamente ubicuo. A partir de las relaciones establecidas con las empresas hibridadoras, las compañías acceden a variedades más sofisticadas que permiten una notable diferenciación. Y esto, sumado a las redes que integran junto con otros productores internacionales es lo que permite desarrollar nuevas variedades de uva que acumulan una mayor cantidad de conocimiento, y que implican productos más complejos. La evolución de la complejidad económica de Moyca es directamente observable en la evolución de su output productivo. Asimismo, la empresa produce actualmente treinta variedades de uva de mesa sin pepita, las cuales corresponden en su mayoría a variedades comunes y, en menor medida, a las variedades especiales y las orgánicas. Sin embargo, la empresa está realizando grandes esfuerzos para el desarrollo de estas últimas variedades, entre las que se destacan aquellas que tienen sabores distintos como la Cotton Candy, una variedad de uva con sabor a algodón de azúcar, u otras con las mismas características pero cuyo cultivo sea totalmente orgánico: variedades producidas sin el uso de pesticidas o productos fitosanitarios, y que además contienen algunas propiedades adicionales que las hagan hacen más beneficiosas para la salud. Aunque, en su mayor parte, las que se producen no son especiales ni orgánicas, la empresa ha demostrado capacidades para introducirlas en su output productivo, lo cual significa que su capacidad computacional ha aumentado; en ese sentido, se ha permitido introducir variedades que tienen un mayor valor añadido y que acumulan una mayor cantidad de información. Estos procesos han permitido asimismo la generación de un mayor valor añadido, y un incremento de la capacidad de remuneración que la actividad de la empresa puede otorgar por cada factor de producción. Esto es consistente con unos niveles de precios de la uva apirena, verificables, tanto en el campo como a la salida de almacén (Tabla I); valores sensiblemente superiores a los que obtienen los productores de uvas tradicionales.

De acuerdo con lo anterior, la crucial decisión que adoptó la empresa en 1995, cuando empezó a producir exclusivamente uva apirena, exigió la visión empresarial, la capacidad de adaptación y el liderazgo para vencer las inercias de los sistemas ante el cambio y la innovación. En este sentido, es destacable que la innovación no solamente le concierne al nuevo producto, sino especialmente a los cambios en los procesos de gestión y en las relaciones de la empresa que han exigido, entre otros retos, la conformación de redes más amplias vinculadas con sus contrapartes internacionales. Sin el trabajo en red, la empresa objeto de estudio y su modelo de negocio no serían viables; en consecuencia, resulta imprescindible vencer las dificultades y las resistencias, que también tienen índole cultural y mental: barreras que están presentes en las empresas y, probablemente en mayor medida, en los sectores rurales de países como Colombia y España.

Tras haber examinado el proceso de crecimiento de la empresa Moyca según la perspectiva de la complejidad económica, se destaca que el aumento del conocimiento incorporado en sus diferentes actividades y procesos, al igual que su transferencia al output productivo, hayan constituido los aspectos determinantes de ese crecimiento. La acumulación de conocimiento no la ha realizado Moyca por sí sola, sino que ha precisado de la formación de redes con otros productores y con las empresas hibridadoras. Además, Moyca participa en otras relaciones que involucran a tres universidades, al igual que entabla lazos con empresas de biofarmacia para el desarrollo de productos enriquecidos y beneficiosos para la salud, junto con la obtención de compuestos a partir de la uva, joint ventures con empresas de zumos y compañías dedicadas a actividades como el desarrollo de aditivos de uva para la industria.

Acorde con la perspectiva de la complejidad económica, los procesos de especialización, diversificación y formación de redes con otras empresas e instituciones, las cuales a su vez han crecido y evolucionado de manera simultánea y sostenida, han permitido la acumulación de conocimiento e información en la empresa. Así, ha sido posible impulsar el crecimiento económico y la evolución de Moyca, que pasó de ser una pequeña empresa familiar a transformarse en una empresa internacionalizada y de grandes dimensiones. 


\section{Referencias}

Barder, O. (2012). Development and complexity. Center of Global Development in Europe.

Beinhocker, E. D. (2006). Origin of wealth: evolution, complexity, and the radical remaking of economics. Boston, MA: Harvard Business Review Press.

Benham-Hutchins, M. y Clancy, T. R. (2010). Social networks as embedded complex adaptive systems. Journal of Nursing Administration, 40(9).

Coase, R. H. (1937). The nature of the firm. Economica, 4(16), 386-405. https://doi.org/10.2307/2626876.

Gell-Mann, M. (1995). What is complexity? Remarks on simplicity and complexity by the Nobel Prize-winning author of The Quark and the Jaguar. Complexity, I(I), I6-19. https://doi.org/10.1002/cplx.6130010105

Gell-Mann, M. (1988). Simplicity and complexity in the description of nature. Engineering and Science, $5 I(3), 2-9$.

Hausmann, R., Cheston, T. y Santos, M. A. (2015). La complejidad económica de Chiapas: análisis de capacidades y posibilidades de diversificación productiva. Cambridge, MA: Center for International Development at Harvard University.

Hausmann, R., Hidalgo, C. A., Bustos, S., Coscia, M., Chung, S., Jiménez, J., . . Yildririm, M. A. (2013). The atlas of economic complexity: mapping paths to prosperity. Updated edition. Cambridge, MA: The MIT Press.

Hausmann, R., Hwang, J. y Rodrik, D. (2007). What you export matters. Journal of Economic Growth, I2(I), I-25. ht tps://doi.org/I0.1007/sio887-006-9009-4

Hausmann, R. y Klinger, B. (2006). Structural Transformation and Patterns of Comparative Advantage in the Product Space (SSRN Scholarly Paper No. ID 939646). Rochester, NY: Social Science Research Network.

Hidalgo, C. A. (2015). Why information grows: the evolution of order, from atoms to economies. New York, NY: Basic Books.

Hidalgo, C. A., Hausmann, R. y Dasgupta, P. S. (2009). The building blocks of economic complexity. Proceedings of the National Academy of Sciences of the United States of America, I06(26), 10570-10575.

Hidalgo, C. A., Klinger, B., Barabási, A. L. y Hausmann, R. (2007). The product space conditions the development of nations. Science, 3I7(5837), 482-487. https://doi.org/IO.II26/science.II4458I

Imbs, J. y Wacziarg, R. (2003). Stages of diversification. American Economic Review, 93(I), 63-86. https://doi.org/ı0 $.1257 / 000282803321455160$

Martínez Carazo, P. C. (2006). El método de estudio de caso: estrategia metodológica de la investigación científica. Pensamiento E. Gestión, (20), 165-193.

Prebisch, R. (1986). El desarrollo económico de la América Latina y algunos de sus principales problemas. Desarrollo Económico, 26(103), 479-502. https://doi.org/10.2307/3466824

Rodrik, D. (2005). Políticas de diversificación económica. Revista de la CEPAL, 87, 7-23.

\section{Notas}

* Artículo de investigación.

Licencia Creative Commons BY-4.० 